For the case of hydrostatic pressure,

$$
N(t)=b^{2}-t^{2}, \quad|t|<b ; \quad N(t)=0, \quad|t|>b, \quad T(t)=0, \quad \text { all } t,
$$

we can take

$$
\Phi_{3}(\zeta)=b^{2} \Phi_{1}(\zeta)-\Phi_{2}(\zeta), \quad \Psi_{3}(\zeta)=b^{2} \Psi_{1}(\zeta)-\Psi_{2}(\zeta),
$$

or we can proceed as in the previous examples.

\title{
REFERENCES
}

1. N. I. Muskhelishvili, Some basic problems of the mathematical theory of elasticity, 3rd ed., Moscow, 1949. (Translated by J. R. M. Radok, P. Noordhoff Ltd., Gronigen, Holland, 1953).

2. R. Tiffen, Solution of two dimensional elastic problems by conformal mapping to a half-plane, Quart. J. Mech. Appl. Math. 5, 352-360 (1952)

\section{ON THE INTEGRATION METHODS OF BERGMAN AND LE ROUX*}

BY J. B. DIAZ1 AND G. S. S. LUDFORD² (University of Maryland)

Introduction. In a previous note [7] a correspondence was found between the representation of solutions, $u(x, y)$, of the linear hyperbolic differential equation

$$
L(u) \equiv u_{x y}+a(x, y) u_{x}+b(x, y) u_{y}+c(x, y) u=0,
$$

in the forms

$$
u(x, y)=2 \int_{0}^{1} E(x, y, t) f\left[\frac{1}{2} x\left(1-t^{2}\right)\right] \frac{d t}{\left(1-t^{2}\right)^{1 / 2}}
$$

and

$$
u(x, y)=\int_{0}^{x} U(x, y, \alpha) g(\alpha) d \alpha .
$$

The first representation is due to Bergman $[3,4,6]$ and the second to Le Roux [1]. In Eq. (2), $E(x, y, t)$ is the even part of a solution for $-1 \leq t \leq 1$ of

$$
\left(1-t^{2}\right)\left(E_{y t}+a E_{\imath}\right)-\frac{1}{t}\left(E_{y}+a E\right)+2 x L(E)=0,
$$

such that, for $x \neq 0$,

$$
\frac{\left(1-t^{2}\right)^{1 / 2}\left(E_{y}+a E\right)}{x t}
$$

is continuous for $t=0$, and tends to zero for each $(x, y)$ as $t$ approaches +1 ; in Eq. (3), $U(x, y, \alpha)$ is a one-parameter family of solutions of Eq. (1) satisfying the characteristic condition

$$
\frac{\partial U}{\partial y}+a U=0 \text { on } \quad x=\alpha .
$$

*Received February 18, 1956.

'The research of this author was supported by the United States Air Force through the Office of Scientific Research.

${ }^{2}$ The work of this author was sponsored by the Office of Ordnance Research, U. S. Army, under Contract DA-36-034-ORD-1486. 
The correspondence in question is given by

$$
\left\{\begin{aligned}
g(\alpha) & =f\left(\frac{1}{2} \alpha\right), \\
U(x, y, \alpha) & =\frac{E\left[x, y,\left(\frac{x-\alpha}{x}\right)^{1 / 2}\right]}{[\alpha(x-\alpha)]^{1 / 2}} .
\end{aligned}\right.
$$

However, it has the disadvantage that to functions $E(x, y, t)$, continuous at $t=0$ and $t=1$, there may correspond functions $U(x, y, \alpha)$ which are singular for $\alpha=x$ and $\alpha=0$. The purpose of the present note is to give a second correspondence, whenever $f(0)=0$, which avoids this difficulty. The modification necessary when $f(0) \neq 0$ will also be given, and an example discussed.

An alternative correspondence. An alternative to Eqs. (7) is suggested by considering the special case $a=b=c=0$ in Eq. (1), the so-called wave equation when $x$ and $y$ are real. Clearly, see Eqs. (4) and (5), a possible choice for $E$ is then $E(x, y, t) \equiv 1$, and a possible choice for $U$ is $U(x, y, \alpha) \equiv 1$, see Eqs. (1) and (6). With these choices one obtains the same function $u(x, y)$ in Eqs. (2) and (3) provided $f$ and $g$ satisfy

$$
2 \int_{0}^{1} f\left[\frac{1}{2} x\left(1-t^{2}\right)\right] \frac{d t}{\left(1-t^{2}\right)^{1 / 2}}=\int_{0}^{x} g(\alpha) d \alpha .
$$

This last equation may be considered as an integral equation for $f$ once $g$ is given. To every ${ }^{3}$ continuous $g$ there corresponds a continuous $f$ with $f(0)=0$. For on setting $\lambda=x\left(1 t^{2}\right)$ in the integral on the left one obtains

$$
\int_{0}^{x} \frac{f\left(\frac{1}{2} \lambda\right)}{\lambda^{1 / 2}} \cdot \frac{d \lambda}{(x-\lambda)^{1 / 2}}=\int_{0}^{x} g(\alpha) d \alpha,
$$

which is an Abel integral equation for $f\left(\frac{1}{2} \lambda\right) / \lambda^{3}$. Its continuous solution [2] is

$$
f\left(\frac{1}{2} x\right)=\frac{x^{1 / 2}}{\pi} \int_{0}^{x} \frac{g(\beta) d \beta}{(x-\beta)^{1 / 2}},
$$

and clearly $f(0)=0$.

On the other hand Eq. (8) is an integral equation for $g$ once $f$ is given. To every ${ }^{4}$ continuously differentiable $f$ with $f(0)=0$ there corresponds a continuous $g$. For clearly if such a $g$ exists it is given by

$$
g(x)=\int_{0}^{1} f^{\prime}\left[\frac{1}{2} x\left(1-t^{2}\right)\right]\left(1-t^{2}\right)^{1 / 2} d t
$$

and it is easily verified by integration that when $f(0)=0$ this $g$ satisfies Eq. (8).

This correspondence between $f$ and $g$, expressed by Eqs. (9) and (10), is not one-toone, since for example there are continuous functions $g$ for which $f$ is not continuously

'In Bergman's work $f$ is required to be at least continuously differentiable, while Le Roux needs $g$ to be just continuous.

'In Bergman's work $f$ is required to be at least continuously differentiable, while Le Roux needs $g$ to be just continuous. 
differentiable ${ }^{5}$. However, there is in fact such a correspondence between continuous functions $g$ and continuous functions $f$, with $f(0)=0$, for which the integral on the left side of $\mathrm{Eq}$. (8) possesses a continuous $x$-derivative. When this correspondence is used, Eq. (10) is replaced by

$$
g(x)=2 \frac{d}{d x} \int_{0}^{1} f\left[\frac{1}{2} x\left(1-t^{2}\right)\right] \frac{d t}{\left(1-t^{2}\right)^{1 / 2}},
$$

and corresponding (trivial) changes must be made in the sequel. Also there is a one-toone correspondence between analytic functions $f$ and $g$ with $f(0)=0$.

It will now be shown that in the general case of Eq. (1) a correspondence between $E$ and $U$ may be chosen so as to preserve the same correspondence between $f$ and $g$. If for a given continuously differentiable $f$ with $f(0)=0$, a continuous function $g$ is determined by means of Eq. (10), then $f$ may be expressed in terms of $g$ by Eq. (9). Inserting this expression for $f$ into Eq. (2), after the change of integration variable $\lambda=x\left(1-t^{2}\right)$ has been made in the latter, one has

$$
u(x, y)=\frac{1}{\pi} \int_{0}^{x} E\left(x, y,\left(\frac{x-\lambda}{x}\right)^{1 / 2}\right)\left[\int_{0}^{\lambda} \frac{g(\beta) d \beta}{(\lambda-\beta)^{1 / 2}}\right] \frac{d \lambda}{(x-\lambda)^{1 / 2}} .
$$

The integral on the right may be transformed by means of Dirichlet's inversion formula into

$$
\frac{1}{\pi} \int_{0}^{x} g(\beta)\left[\int_{\beta}^{x} E\left(x, y,\left(\frac{x-\lambda}{x}\right)^{1 / 2}\right) \frac{d \lambda}{(x-\lambda)^{1 / 2}(\lambda-\beta)^{1 / 2}}\right] d \beta,
$$

which has the form of the right member of Eq. (3) with

$$
\begin{aligned}
U(x, y, \beta) & =\frac{1}{\pi} \int_{\beta}^{x} E\left(x, y,\left(\frac{x-\lambda}{x}\right)^{1 / 2}\right) \frac{d \lambda}{(x-\lambda)^{1 / 2}(\lambda-\beta)^{1 / 2}}, \\
& =\frac{2}{\pi} \int_{0}^{\pi / 2} E\left(x, y,\left(\frac{x-\beta}{x}\right)^{1 / 2} \cos \theta\right) d \theta,
\end{aligned}
$$

where $\lambda=x \sin ^{2} \theta+\beta \cos ^{2} \theta$.

It is easily shown ${ }^{6}$ that if $E(x, y, t)$ satisfies Eq. (4) for $t \neq 0$, then for each $\beta$ the function $U(x, y, \beta)$, defined by Eq. (11), satisfies Eq. (1) when $x \neq 0, \beta$. In addition, if $E(x, y, t)$ satisfies the condition (5) (which implies $E_{\nu}+a E \rightarrow 0$ as $t \rightarrow 0$ ) then $U(x, y, \beta)$ satisfies (6) on $x=\beta$. Unlike the previous correspondence (7), the present one has the property that to functions $E(x, y, t)$ which are continuous at $t=0$ and $t=1$, there correspond functions $U(x, y, \beta)$ which are continuous for $\beta=x$ and $\beta=0$.

In order to invert Eq. (11a) so as to obtain $E$ in terms of $U$ this equation may be considered, for fixed $x$ and $y$, as an Abel integral equation for $E$ with variable $\beta$. Thus

$$
E\left(x, y,\left(\frac{x-\gamma}{x}\right)^{1 / 2}\right)=U(x, y, x)-(x-\gamma)^{1 / 2} \int_{\gamma}^{x} \frac{U_{\beta}(x, y, \beta)}{(\beta-\gamma)^{1 / 2}} d \beta
$$

5To $g(x)=0$ for $x<x_{0}$ and $g(x)=\left(x-x_{0}\right)^{\alpha}$ for $x \geq x_{0}$, where $\alpha>0$, there corresponds $f(x)=0$ for $x<x_{0}$ and $f(x / 2)=A x^{1 / 2}$. $\left(x-x_{0}\right)^{\alpha+1 / 2}$ where $A$ is a non-zero constant. If $\alpha<\frac{1}{2}$, then $f$ is not differentiable at $x=x_{0}$.

If in particular $E$ is analytic and even in $t$, then $U$ is analytic for $x \neq 0$. 
On setting $\gamma=x\left(1-t^{2}\right)$ and changing the integration variable from $\beta$ to $\xi$, where $\beta=$ $x\left(1-\xi t^{2}\right)$, one finds

$$
E(x, y, t)=U(x, y, x)-x t^{2} \int_{0}^{1} \frac{U_{\beta}\left[x, y, x\left(1-\xi t^{2}\right)\right]}{(1-\xi)^{1 / 2}} d \xi .
$$

Extension of the correspondence. So far only functions $f$ have been considered for which $f(0)=0$. When $f(0) \neq 0$ an extra term arises in Eq. (3) if the transformation of the previous section is applied to Eq. (2). Thus the latter may be written

$$
u(x, y)=u_{1}(x, y)+u_{2}(x, y) \text {, }
$$

where

$$
\begin{aligned}
& u_{1}(x, y)=2 f(0) \int_{0}^{1} E(x, y, t) \frac{d t}{\left(1-t^{2}\right)^{1 / 2}}, \\
& u_{2}(x, y)=2 \int_{0}^{1} E(x, y, t) F\left[\frac{1}{2} x\left(1-t^{2}\right)\right] \frac{d t}{\left(1-t^{2}\right)^{1 / 2}},
\end{aligned}
$$

and $F\left[\frac{1}{2} x\left(1-t^{2}\right)\right]=f\left[\frac{1}{2} x\left(1-t^{2}\right)\right]-f(0)$. The preceding analysis may be applied to $u_{2}(x, y)$, since $F(0)=0$. Thus $u_{2}(x, y)$ may be written in the form of Eq. (3) where $U(x, y, \alpha)$ is given by Eqs. (11) and, according to Eq. (10),

$$
\begin{aligned}
g(x) & =\int_{0}^{1} F^{\prime}\left[\frac{1}{2} x\left(1-t^{2}\right)\right]\left(1-t^{2}\right)^{1 / 2} d t, \\
& =\int_{0}^{1} f^{\prime}\left[\frac{1}{2} x\left(1-t^{2}\right)\right]\left(1-t^{2}\right)^{1 / 2} d t .
\end{aligned}
$$

The remaining term $u_{1}(x, y)$ may be written

$$
\begin{aligned}
u_{1}(x, y) & =2 f(0) \int_{0}^{\pi / 2} E(x, y, \cos \theta) d \theta, \\
& =\pi f(0) U(x, y, 0),
\end{aligned}
$$

in view of Eq. (11b). Hence finally

$$
u(x, y)=\pi f(0) U(x, y, 0)+\int_{0}^{x} U(x, y, \alpha) g(\alpha) d \alpha .
$$

The first term on the right hand side of this equation is clearly a solution of Eq. (1) since $U(x, y, \alpha)$ is for all $\alpha$.

An alternative form of Eq. (14) is obtained on writing

$$
w(x)=2 \int_{0}^{1} f\left[\frac{1}{2} x\left(1-t^{2}\right)\right] \frac{d t}{\left(1-t^{2}\right)^{1 / 2}},
$$

so that $w(0)=\pi f(0)$ and $g(\alpha)=w^{\prime}(\alpha)$, see Eq. (8). For then on integration by parts Eq. (14) becomes

$$
\begin{aligned}
u(x, y) & =\pi f(0) U(x, y, 0)+[U(x, y, \alpha) w(\alpha)]_{\alpha=0}^{\alpha=x}-\int_{0}^{x} U_{\alpha}(x, y, \alpha) w(\alpha) d \alpha, \\
& =U(x, y, x) w(x)-\int_{0}^{x} U_{\alpha}(x, y, \alpha) w(\alpha) d \alpha .
\end{aligned}
$$


Example. Eq. (16) is the form of solution obtained by von Mises and Schiffer [5, especially p. 258] in their discussion of Bergman's integration method as applied to the equation satisfied by the "modified" stream function of a compressible flow in the hodograph plane. This equation is a special case of Eq. (1) $\operatorname{with}^{7} a=b=0, c=f(x+y)$. For the $U(x, y, \alpha)$ of the present paper they find

$$
U(x, y, \alpha)=1+\sum_{n=1}^{\infty} G_{n}(x+y) \frac{(-1)^{n}(x-\alpha)^{n}}{2^{n} n !},
$$

where

$$
G_{0}=1, \quad G_{n+1}^{\prime}=G_{n}^{\prime \prime}+f G_{n},
$$

and the prime denotes differentiation with respect to the argument $x+y$.

The same results are obtained with Bergman's representation, Eq. (2), with [4, especially p. 24]

$$
E(x, y, t)=1+\sum_{n=1}^{\infty} t^{2 n} x^{n} Q_{n}(x+y)
$$

when $w$ and $f$ are related by Eq. (15). Here $Q_{n}$ satisfies

$$
Q_{0}=1, \quad(2 n+1) Q_{n+1}^{\prime}+Q_{n}^{\prime \prime}+f Q_{n}=0 .
$$

It is easily verified that the functions $U(x, y, \alpha)$ and $E(x, y, t)$ given by Eqs. (17) and (19) are in fact connected by the general formulas (11b) and (12). For example, with $E(x, y, t)$ as in Eq. (19)

$$
\begin{aligned}
\int_{0}^{\pi / 2} E\left(x, y,\left(\frac{x-\beta}{x}\right)^{1 / 2} \cos \theta\right) d \theta & =\frac{\pi}{2}+\sum_{n=1}^{\infty}(x-\beta)^{n} Q_{n} \int_{0}^{\pi / 2} \cos ^{2 n} \theta d \theta, \\
& =\frac{\pi}{2}+\frac{\pi}{2} \sum_{n=1}^{\infty} \frac{(2 n-1)(2 n-3) \cdots 1}{2^{n} n !}(x-\beta)^{n} Q_{n}, \\
& =\frac{\pi}{2}\left[1+\sum_{n=1}^{\infty} \frac{(-1)^{n}(x-\beta)^{n}}{2^{n} n !} G_{n}\right],
\end{aligned}
$$

and $G_{n}=(-1)^{n}(2 n-1)(2 n-3) \cdots 1 . Q_{n}$ clearly satisfies Eqs. (18) when $Q_{n}$ satisfies Eqs. (20).

\section{REFERENCES}

1. J. Le Roux, Sur les intérales des équations linéaires aux derivées partielles du second ordre à deux variables indépendantes, Ann. Ec. Norm. Sup. (3), 12, 227-316 (1895)

2. M. Bôcher, An introduction to the study of integral equations, Cambridge, 1909, pp. 8-11

3. S. Bergman, Zur Theorie der Functionen, die eine lineare partielle Differentialgleichung befriedigen, Mathematicheskii Sbornik, New Series 2, 1169-1198 (1937)

4. S. Bergman, Operator methods in the theory of compressible fluids, Proc. Symp. in Appl. Math. (A.M.S.), $1,19-40$ (1949)

5. R. von Mises and M. Schiffer, On Bergman's integration method in two-dimensional compressible fluid flow, Advances in Applied Mechanics (Academic Press), 1, 249-285 (1948)

6. S. Bergman, Operatorenmethoden in der Gasdynamik, Z. Angew. Math. u. Mech. 32, 33-45 (1952)

7. J. B. Diaz and G. S. S. Ludford, On two methods of generating solutions of linear partial differential equations by means of definite integrals, Quart. Appl. Math. 12, 422-427 (1955)

'Explicitly $x=\frac{1}{2}(\lambda+i \theta), y=\frac{1}{2}(\lambda-i \theta)$. 